This item was submitted to Loughborough's Research Repository by the author.

Items in Figshare are protected by copyright, with all rights reserved, unless otherwise indicated.

\title{
Surface measurement errors using commercial scanning white light interferometers
}

PLEASE CITE THE PUBLISHED VERSION

PUBLISHER

(c) IOP PUBLISHING

LICENCE

CC BY-NC-ND 4.0

REPOSITORY RECORD

Gao, F., Richard K. Leach, Jon N. Petzing, and Jeremy M. Coupland. 2019. "Surface Measurement Errors Using Commercial Scanning White Light Interferometers". figshare. https://hdl.handle.net/2134/3633. 
This item was submitted to Loughborough's Institutional Repository by the author and is made available under the following Creative Commons Licence conditions.

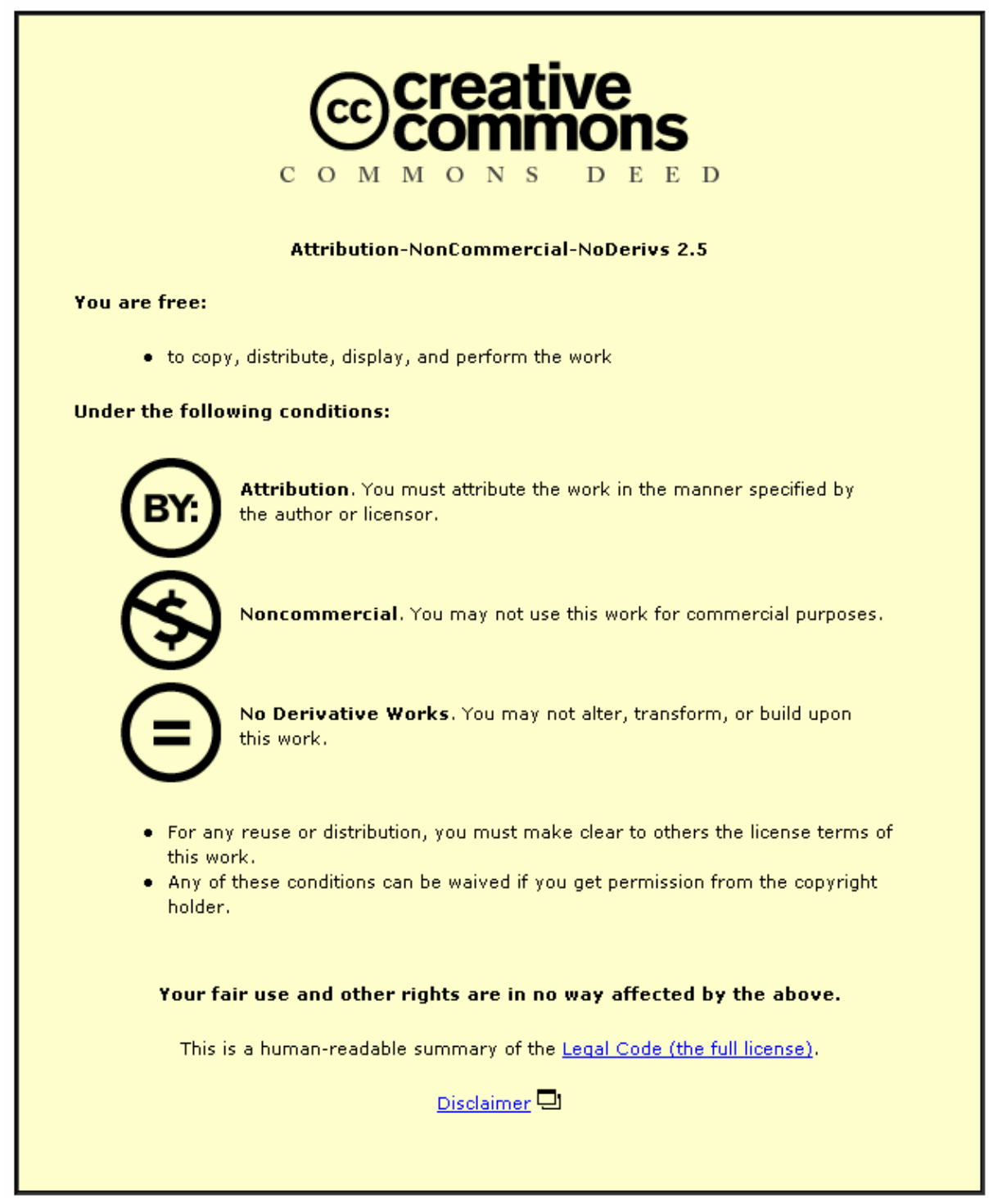

For the full text of this licence, please go to: http://creativecommons.org/licenses/by-nc-nd/2.5/ 


\title{
Surface Measurement Errors using Commercial Scanning White Light Interferometers
}

\author{
F Gao ${ }^{1}$, R K Leach ${ }^{2}$, J Petzing ${ }^{1}$ and J M Coupland ${ }^{1}$ \\ ${ }^{1}$ Wolfson School of Mechanical and Manufacturing Engineering, Loughborough University, \\ Loughborough, Leicestershire LE11 3TU, UK \\ ${ }^{2}$ Industry \& Innovation Division, National Physical Laboratory, Hampton Road, Teddington, \\ Middlesex TW11 0LW, UK
}

F.Gao@lboro.ac.uk

\begin{abstract}
:
This paper examines the performance of commercial scanning white light interferometers in a range of measurement tasks. A step height artefact is used to investigate the response of the instruments at a discontinuity, while gratings with sinusoidal and rectangular profiles are used to investigate the effects of surface gradient and spatial frequency. Results are compared with measurements made with tapping mode atomic force microscopy and discrepancies are discussed with reference to error mechanisms put forward in the published literature. As expected it is found that most instruments report errors when used in regions close to a discontinuity or those with a surface gradient that is large compared to the acceptance angle of the objective lens. Amongst other findings, however, we report systematic errors that are observed when the surface gradient is considerably smaller. Although these errors are typically less than the mean wavelength they are significant compared to the instrument resolution and indicate that current scanning white light interferometers should be used with some caution if sub-wavelength accuracy is required.
\end{abstract}

Key words: White light interferometry, measurement errors, surface roughness measurement, multiple scattering effect.

PACS code: 42.25.Kb (Coherence), 42.25.Fx (Diffraction and scattering) 42.25.Hz (Interference), 47.50.Ef (Measurements)

\section{Introduction}

Scanning white light interferometry (SWLI) is now an established method to measure precision surfaces [1-5]. In comparison with stylus profilometry and scanning probe microscopy, SWLI presents significant advantages. It is an optical, non-contacting technique and as such can be used to measure the profile of delicate membranes or soft materials without risk of damage. It is also significantly faster than confocal or near field microscopes since SWLI only requires a scan in a single (vertical) direction. SWLI shares many characteristics with its close relative, phase shifting interferometry (PSI), but differs essentially in the use of an extended polychromatic source that allows the fringe order to be identified unambiguously and results in a virtually unbounded measurement range. As an interferometric technique, the vertical resolution of SWLI is limited by the precision to which 
the phase of the reflected signal can be identified and is typically around one thousandth of the mean wavelength (ie sub-nanometre). As a high power microscope, SWLI promises lateral resolution up to the Rayleigh diffraction limit, which is typically around $0.5 \mu \mathrm{m}$ for objectives of large numerical aperture (NA).

In contrast with PSI, a stable source is not exploited in most SWLI instrumentation and calibration of the scanning mechanism is required to ensure accuracy. For the measurement of constrained surfaces such as step heights and gauge blocks this is sufficient to provide traceability. However, the popularity of SWLI has led to its use in circumstances that are far removed from traditional gauge block interferometry and SWLI manufacturers have been quick to cater for these demands by including, for example, specialised software to estimate areal surface texture parameters and film thickness $[6,7]$. In these cases, the accuracy of SWLI is much more difficult to define.

In 1990, Hillmann questioned the accuracy of surface measurements obtained by optical methods [8]. He reported that the results measured on a roughness standard by optical methods present significant deviations with reference to the results from stylus profilometry. This work was of great concern to practicing experimentalists and has provoked many more to document problems encountered with SWLI in surface measurement [8-13]. It is fair to point out that most of the problems cited in the literature have been observed when a surface gradient is large compared to the NA. Most samples, however, will have regions where geometry, surface roughness or debris means that this is not the case, and anomalous measurements can result. We briefly summarise the errors that are observed in the following paragraphs.

The batwing effect is a well known example of an error that is observed around a step discontinuity especially for the case of a step height that is less than the coherence length of the light source $[10,11]$. This problem is called the batwing effect, because of the shape of the error (see for example Figure 15) and it is usually explained as the interference between reflections of waves normally incident on the top and bottom surfaces following diffraction from the edge. Interestingly, an SWLI does not give the correct surface height at the positions close to the step even if the step height is significantly greater than the coherence length. This can be thought of as a phase change caused purely by the way the optical field interacts (diffracts) around the discontinuity. In this case the measurement error is small but still significant compared to the instrument resolution.

On some early SWLI systems, stepped artefacts have been reported when measuring perfectly flat objects [12]. Errors of this kind are commonly referred to as ghost steps. These usually correspond to a $2 \pi$ phase jump or a surface height error of around half the mean wavelength. More generally phase jumps of this magnitude are referred to as $2 \pi$ errors and can be thought of as a misclassification of fringe order $[13,14]$. In this case the error is due to a field dependent dispersion that is due to the geometry of Mirau interference objectives [12]. If spatial phase unwrapping algorithms are implemented the effect can be minimised and the use of matched objectives in reference and object arms of a Twyman-Green interferometer is also reported to reduce the effect.

A similar dispersive effect makes SWLI instrumentation sensitive to surface gradient [15]. Tilt dependent dispersion is often the cause of $2 \pi$ errors in SWLI measurements even when the tilt is small compared to the NA of the objective. If errors of this kind are present then $2 \pi$ errors can appear, for example, at regular intervals on regular sinusoidal profiles (see for example Figure 10a). A combination of field and tilt dependent dispersion is also responsible for errors of similar appearance to the batwing effect and was recently reported by Lehmann [16]. In contrast with regular batwing errors, the presence of these phase jumps depends systematically on position and generally increases in severity toward the edge of the field of 
view. This effect also depends strongly on the polarity of the discontinuity. It is noted that (depending on the height retrieval algorithm used) in extreme cases the dispersive batwing effect can result in errors that propagate and result in a corresponding error in step height measurement (see for example Figure 5).

Dispersive effects of the kind mentioned are clearly a function of the quality of the optical system (they are artefacts of chromatic aberration), however, the optical properties of the surface to be measured are also a potential source of error. It is well known that different materials exhibit different phase changes on reflection, and depending on the processing algorithms used, these will affect the surface height measurement $[13,17]$. Phase changes are typically less than 45 degrees (corresponding to surface height errors of less than $30 \mathrm{~nm}$ ) but can combine with dispersive effects to give $2 \pi$ errors as discussed previously. Clearly, this type of error is only a problem when two or more materials with different optical properties are present in a sample.

Finally it is important to note that surface roughness plays a significant role in measurement quality when using SWLI instrumentation. Many researchers have found that estimates of surface roughness derived from SWLI measurements differ significantly from other measurement techniques $[8,18-21]$. The surface roughness is generally over estimated by SWLI and this can be attributed to multiple scattering. Although it may be argued that the local gradients of rough surfaces exceed the limit dictated by the NA of the objective and, therefore, would be classified as beyond the capability of SWLI instrumentation, measured values with high signal to noise ratio are often reported in practice. If for example, a silicon V-groove (with an internal angle of 70.52 degrees) is measured, a clear peak is observed at the bottom of the profile due to multiple reflections (scattering) [22]. Although this example is specific to a highly polished V-groove fabricated in silicon it is believed to be the cause for over estimation of surface roughness since a roughened surface can be considered to be made up of randomly oriented grooves with varying internal angles.

The error mechanisms described in the preceding paragraphs and references therein, have either been discussed with reference to a specific instrument or a generic model of SWLI. Although commercial SWLI hardware is very similar, the instruments differ quite significantly in the way that they record and process fringe data. It is clear that the identification of fringe order and the inference of surface topography from fringe data is a highly non-linear process and consequently has a large bearing on the final measurements. Although the basic processing methods used by the manufacturers are outlined in patent and other literature [23-25] the actual algorithms that are used remain uncertain. In this paper we provide a comparison of the performance of commercial SWLI instrumentation for a number of measurement tasks. A step artefact is used to investigate the step response of the instruments, while gratings with sinusoidal and rectangular profiles are used to investigate the effects of surface gradient and spatial frequency. The performance is discussed with reference to the error sources outlined above.

It is noted that the intention of this work was not to appraise the relative performance of commercial SWLI instrumentation, indeed the measurements presented are neither sufficiently controlled nor suitably comprehensive for this task. The aim of our work is to demonstrate and discuss the errors that are frequently observed when commercial instrumentation is applied to the measurement of small scale artefacts. To avoid any attempt form an appraisal from the information presented here, we refer to the instruments by a simple alphabetic label.

\section{Instrumentation}


Tests were made on commercial SWLI instruments provided by commercial SWLI instrument manufactures, and with the areal profilometry equipment within the metrology laboratory at Loughborough University. Measurements made by the commercial instruments were performed by measurement engineers at the company or on the premises of their UK representatives. The commercial SWLI instruments involved are labeled Instrument B, Instrument $C$ and Instrument D. At Loughborough University measurements were made using a SWLI labeled Instrument A which was made by the same manufacture as Instrument B and an atomic force microscope (AFM) in a controlled environment $\left(20{ }^{\circ} \mathrm{C}, 45 \%\right.$ relative humidity). The manufacturer's specifications of these instruments are listed in Table 1.

\begin{tabular}{|c|c|c|c|c|c|c|}
\hline & $\begin{array}{l}\text { Objective } \\
\text { lens }\end{array}$ & NA & $\begin{array}{l}\text { Vertical } \\
\text { resolution } \\
\quad(\mathrm{nm})\end{array}$ & $\begin{array}{c}\text { Rayleigh } \\
\text { resolution } \\
\lambda=600 \mathrm{~nm} \\
(\mu \mathrm{m})\end{array}$ & $\begin{array}{l}\text { Pixel } \\
\text { resolution } \\
\quad(\mu \mathrm{m})\end{array}$ & Repeatability \\
\hline \multirow{2}{*}{ Instrument A } & $10 X$ & 0.30 & 0.1 & 1.0 & $\begin{array}{l}2.15 \\
0.55\end{array}$ & \multirow{3}{*}{$\begin{array}{l}\mathrm{RMS}<0.01 \mathrm{~nm} \\
\text { Step height }<0.1 \%\end{array}$} \\
\hline & $50 X$ & 0.55 & 0.1 & 0.55 & $\begin{array}{l}0.43 \\
0.11\end{array}$ & \\
\hline Instrument B & $50 X$ & 0.55 & 0.1 & 0.55 & $\begin{array}{l}0.43 \\
0.11\end{array}$ & \\
\hline \multirow{2}{*}{ Instrument $\mathrm{C}$} & $5 X$ & 0.12 & 0.1 & 2.31 & $\begin{array}{l}0.98 \\
0.85\end{array}$ & \multirow{2}{*}{$\mathrm{RMS}<0.01 \mathrm{~nm}$} \\
\hline & $50 X$ & 0.55 & 0.1 & 0.55 & $\begin{array}{l}0.38 \\
0.09\end{array}$ & \\
\hline Instrument D & $50 X$ & 0.55 & 0.01 & 0.55 & 0.36 & $\begin{array}{l}\text { RMS }<0.003 \mathrm{~nm} \\
\text { Step height }<0.1 \mathrm{~nm}\end{array}$ \\
\hline $\mathrm{AFM}^{1}$ & - & - & 0.1 & - & - & - \\
\hline
\end{tabular}

${ }^{1}$ Resolution is tip dependent. It is cited for a tapping mode tip with $10 \mathrm{~nm}$ radius, resolution is listed in the table.

${ }^{2}$ The pixel resolution listed on the top line for each objectives were used in the tests. The bottom lines are the maximum achievable pixel resolution.

Table 1. Instruments used in the comparison and their specifications

Most commercial SWLI instrumentation includes a means to remove spurious data. For example Instrument A prompts the user to define a minimum fringe modulation below which measurements are not reported. In addition the user can elect to remove spikes to post-process the data. In all cases the minimum modulation was set as low as possible (1\% on Instrument A) and spike removal and other filtering operations were requested to be turned off.

For Instrument A, Instrument B and Instrument D, the algorithm used in the instruments to detect the position of the envelop peak involves both intensity and phase fitting. Therefore the working mode for these instruments are combined vertical scanning interferometry (VSI) mode and PSI mode. For Instrument C, only VSI mode was available for the instrument we used in the test.

\section{Measurement Artefacts and Evaluation Methods}

Three types of measurement artefacts were used in this study; a calibrated step height and two sets of grooves with approximately sinusoidal and rectangular profiles respectively.

The step artefact is a $1.844 \mu \mathrm{m}$ step (manufactured by VLSI Standard Inc) that was supplied by the manufacture for calibration of Instrument A. In the study this artefact was used to calibrate the Instrument A used at Loughborough whilst the other instruments were 
calibrated by company engineers using their own artefacts and procedures. Figure 1 shows the profile of the step artefact using the AFM. It is noted that, for a white light interferometer with a mean wavelength of $\lambda_{0}=600 \mathrm{~nm}$ and a bandwidth of $\Delta \lambda=400 \mathrm{~nm}$, the coherence length, $\Delta \mathrm{L}\left(\Delta \mathrm{L}=\lambda^{2} / \Delta \lambda\right)$ is approximately $\Delta \mathrm{L}=900 \mathrm{~nm}$. For the step artefact the difference in path length between light scattered by the top and bottom surfaces (around $3.7 \mu \mathrm{m}$ ) is greater than the coherence length and consequently interference effects are expected.

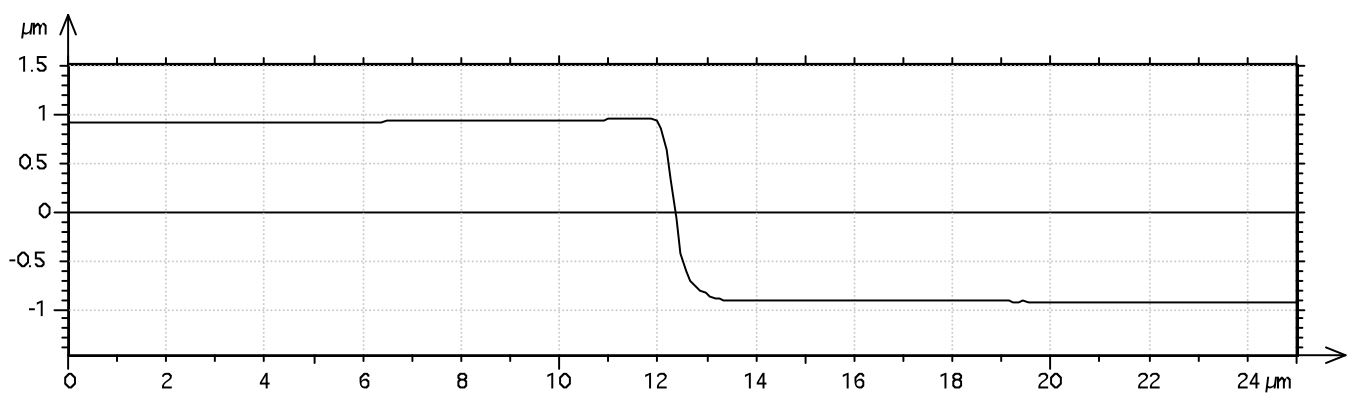

Figure 1. Step profile measured using AFM

The sinusoidal profile artefacts were of gold coated etched glass and were manufactured at the National Physical Laboratory, UK. The sinusoidal artefacts have two periods of $8 \mu \mathrm{m}$ and $25 \mu \mathrm{m}$, and a range of amplitudes as specified in Table 2 . The table also gives the maximum gradient that was calculated from the profiles obtained with the AFM. Measurement of the sinusoidal artefacts provides us with an idea of the dynamic response of SWLI instrumentation in terms of the maximum surface gradient (or slew rate) that can be measured.

\begin{tabular}{ccc}
\hline Pitch $(\mu \mathrm{m})$ & Peak-to-peak amplitude $(\mu \mathrm{m})$ & Maximum gradient (degrees) \\
\hline 25 & 2.720 & 37.2 \\
25 & 0.654 & 5.3 \\
25 & 0.531 & 3.9 \\
25 & 0.134 & 1.1 \\
8 & 0.496 & 17.1 \\
8 & 0.124 & 3.0 \\
8 & 0.068 & 1.5 \\
8 & 0.020 & 0.5 \\
\hline
\end{tabular}

Table 2. Specification of sinusoidal artefacts

To test the lateral resolution of the SWLI instruments an artefact was used that consisted of eight gratings with periods from $1 \mu \mathrm{m}$ to $8 \mu \mathrm{m}$ and an array of star patterns in the central patch. The artefact was fabricated by e-beam lithography on a silicon substrate resulting in a rectangular groove profile. A typical profile measured with the AFM is shown in Figure 2. The etch depth was found to be $184 \mathrm{~nm} \pm 1 \mathrm{~nm}$ using this instrument.

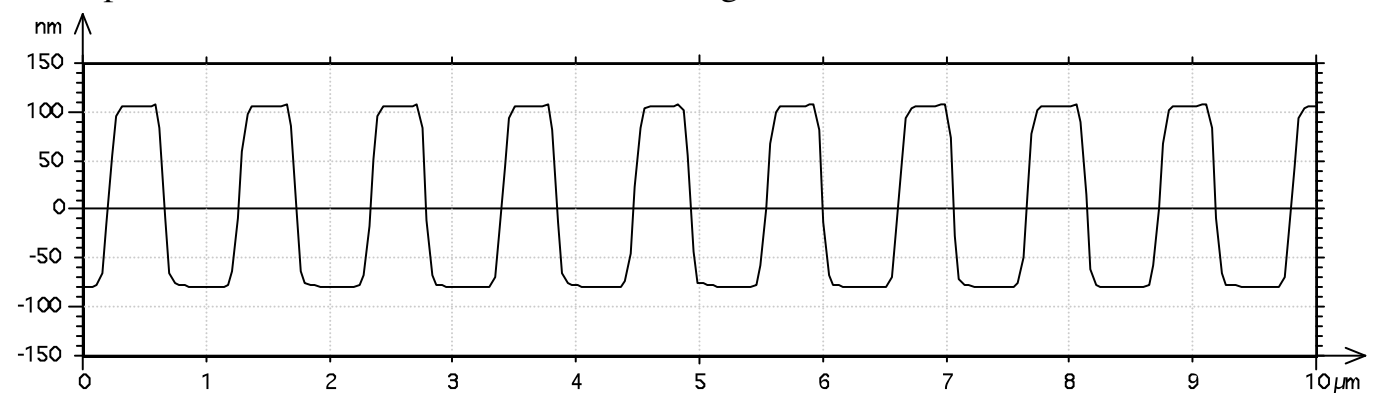

Figure 2. Profile of silicon artefact using the AFM 
It is important to choose an appropriate method to analyse the measurements in order to identify the pertinent characteristics of the surface structure. To make the measurement process comparable we have used the Talymap Gold 4.1 software (Developed by Digital Surf and supplied by Taylor Hobson). This software can import data files from all of the measurement instruments and provides some useful measurement tools. To measure step height we have used the Step Height Measurement Function under the Studies Menu and have selected the ISO 5436-1 method to evaluate the step height measurement results.

For the measurement of peak-to-peak amplitude of the sinusoidal and rectangular profiles we have used a statistical method based on an analysis of the cumulative probability distribution (or Abbott curve) derived from surface height data. For the reasons discussed in Section 2, the SWLI instruments often introduce spurious spikes into the data that typically correspond to half-wavelength or $2 \pi$ errors. The probability analysis is performed on surface height data that is pre-processed (using the least squares plane function) to remove any systematic tilt. The height(s) with the largest probability can be identified as points of maximum gradient on the Abbot curve. Consequently, the Abbott curves corresponding to both sinusoidal profiles have two, clearly defined steps that identify the peak-to-peak amplitude. Examples of Abbott curves for sinusoidal and rectangular profiles are shown in Figure $3 a$ and $3 b$ respectively. Since the main effect of spikes corresponding to $2 \pi$ errors is to smooth the distribution slightly, the method is quite robust unless the measurement noise completely overwhelms the signal.

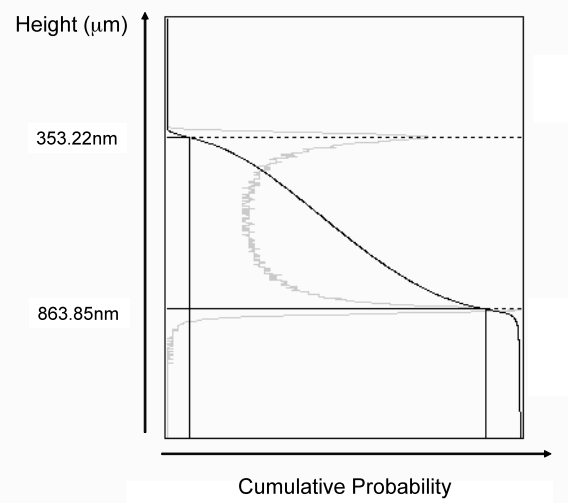

a)

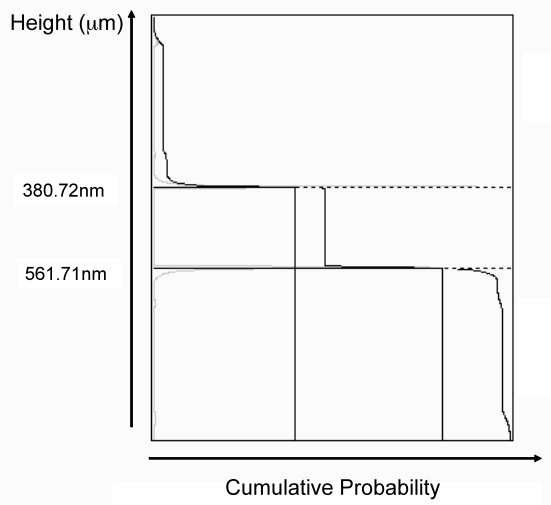

b)

Figure 3 Abbott curves a) sinusoidal and b) rectangular profiles

\section{Results and discussion}

\subsection{Step Height Measurement}

Figure 4 shows typical step height profiles reported by the SWLI instruments using objectives with $\mathrm{NA}=0.55$. It can be seen that the step profile is reasonably well resolved by all the instruments. The figures show a magnified response around the edge and a small batwing effect can be seen most notably on Instrument C. It is noted that this instrument operates purely in VSI mode and we can conclude that full VSI/PSI analysis (see Section 2) provides more precise measurements at a step discontinuity. 


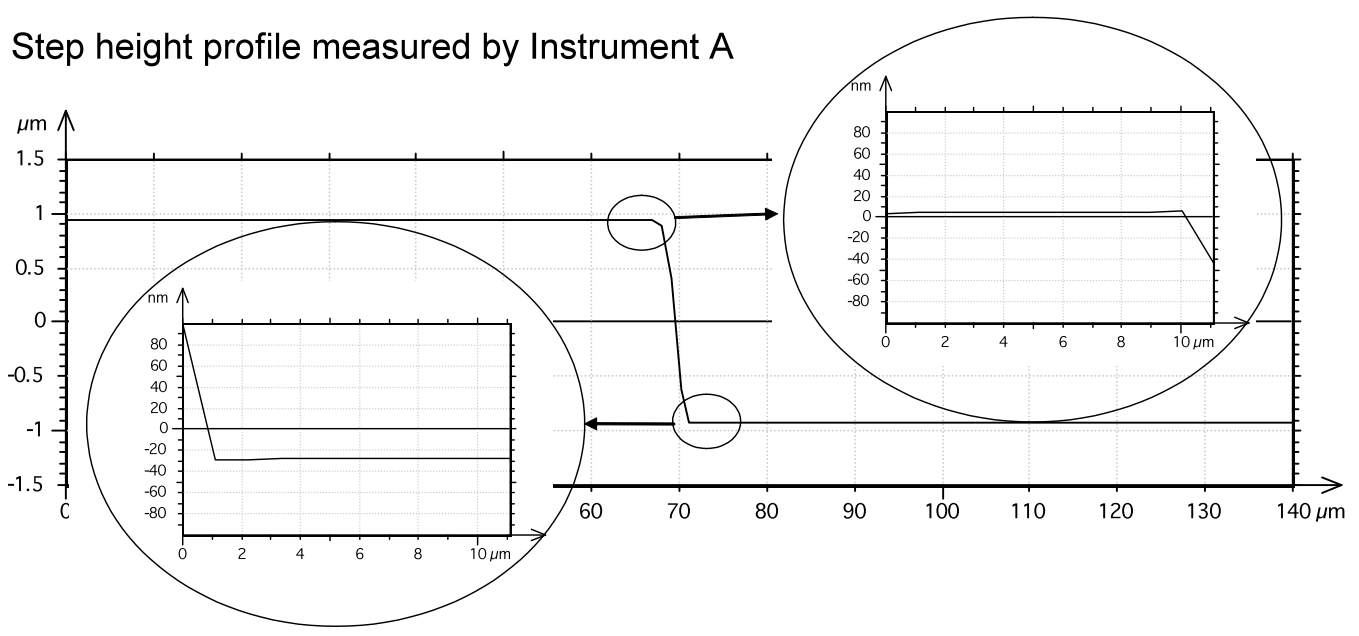

Step height profile measured by Instrument B

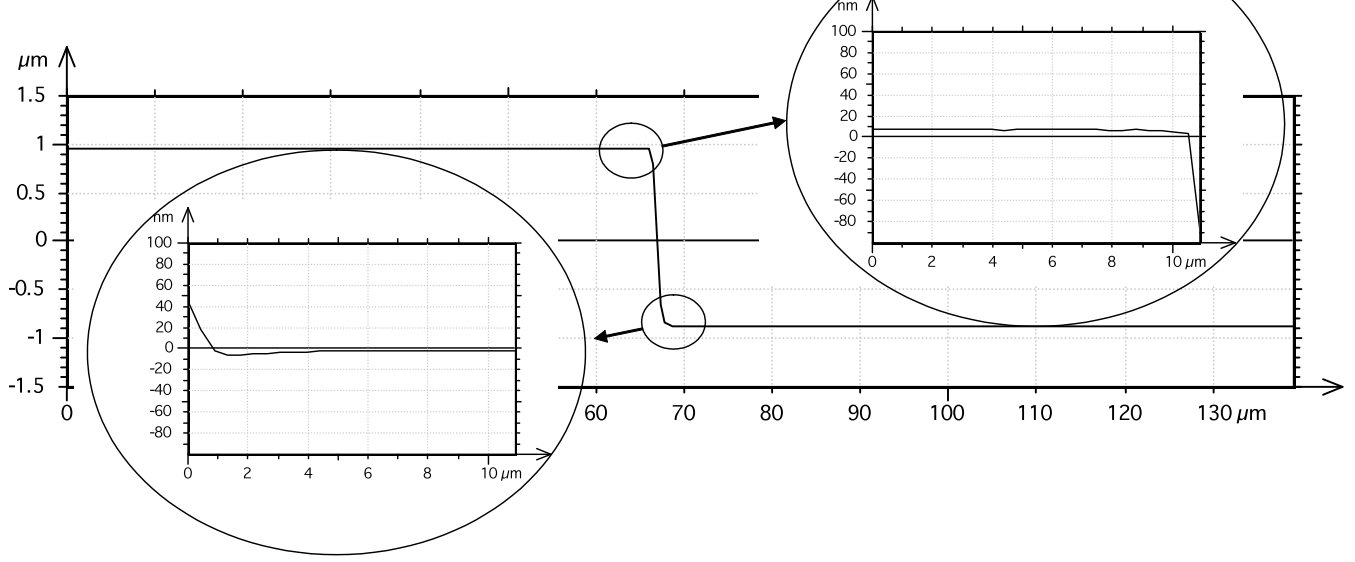

Step height profile measured by Instrument C

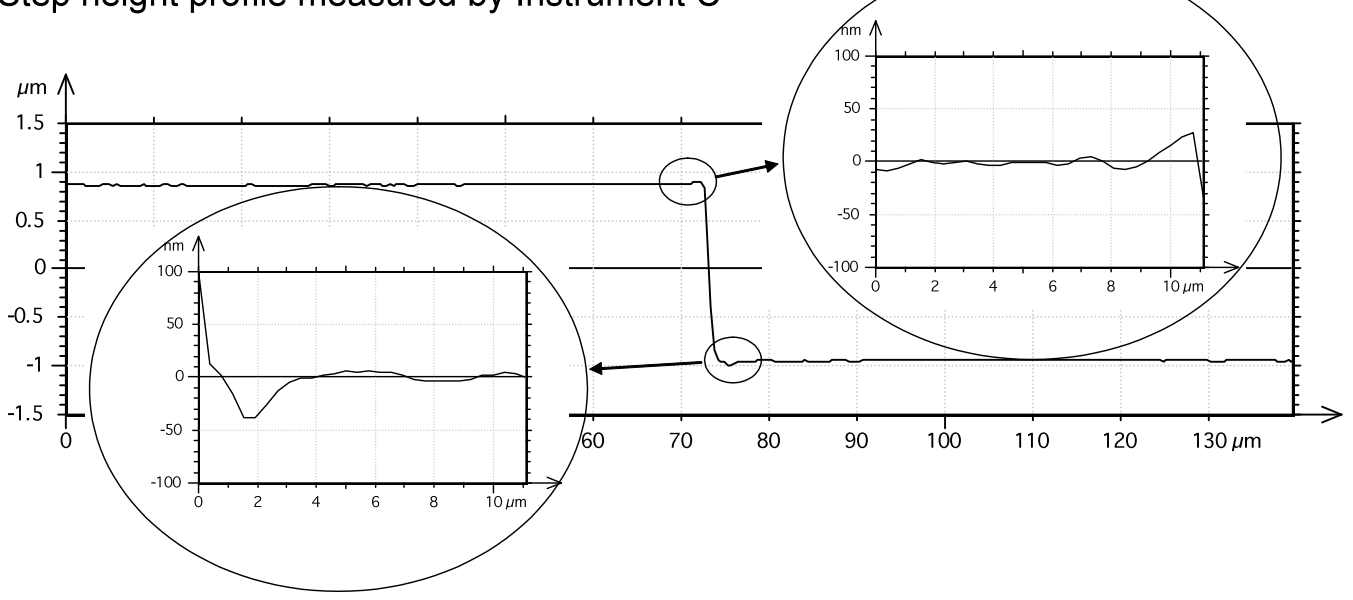




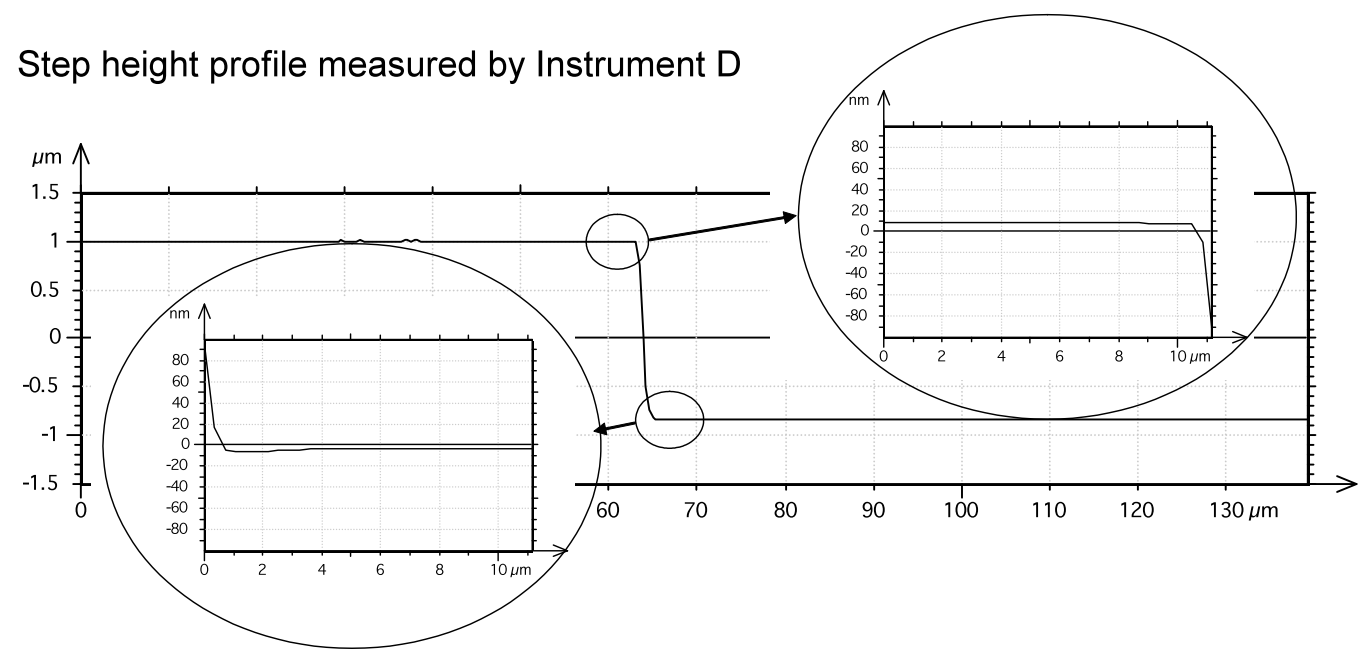

Figure 4. Step profiles obtained with the four commercial instruments

From the line profiles, the step height was calculated according to the procedure described in Section 3 and the results are shown in Table 3. It is noted that Instrument A was calibrated with the same step height artefact that was used in the measurement and it is perhaps not surprising that this instrument returned values that were closest to the nominal value.

\begin{tabular}{ccccccc}
\hline & Instrument A & \multicolumn{1}{c}{ Instrument B } & Instrument C & Instrument D & AFM & $\begin{array}{c}\text { Nominal } \\
\text { Value }\end{array}$ \\
\hline $\begin{array}{c}\text { Step-height } \\
(\mu \mathrm{m})\end{array}$ & 1.844 & $\begin{array}{c}1.842 \\
1.544^{*}\end{array}$ & 1.822 & 1.841 & 1.845 & 1.844 \\
\hline
\end{tabular}

*Initial reading (see text)

Table 3. Step height measurement results

It is important to note that this limited set of data is not representative of the instrument accuracy. It only shows that the measurements reported by Instrument A, Instrument B and Instrument D are within the manufacturer's repeatability specifications (see Table 1) whereas the $22 \mu \mathrm{m}$ discrepancy reported by Instrument $\mathrm{C}$ suggests that the instrument was not correctly calibrated. It is worth mentioning that Instrument B did not perform well initially, reporting a step height of $1.544 \mu \mathrm{m}$. This is a $0.3 \mu \mathrm{m}$ deviation from the nominal value and is likely to correspond to a half-wavelength or $2 \pi$ error. The profile of this measurement is shown in Figure 5.

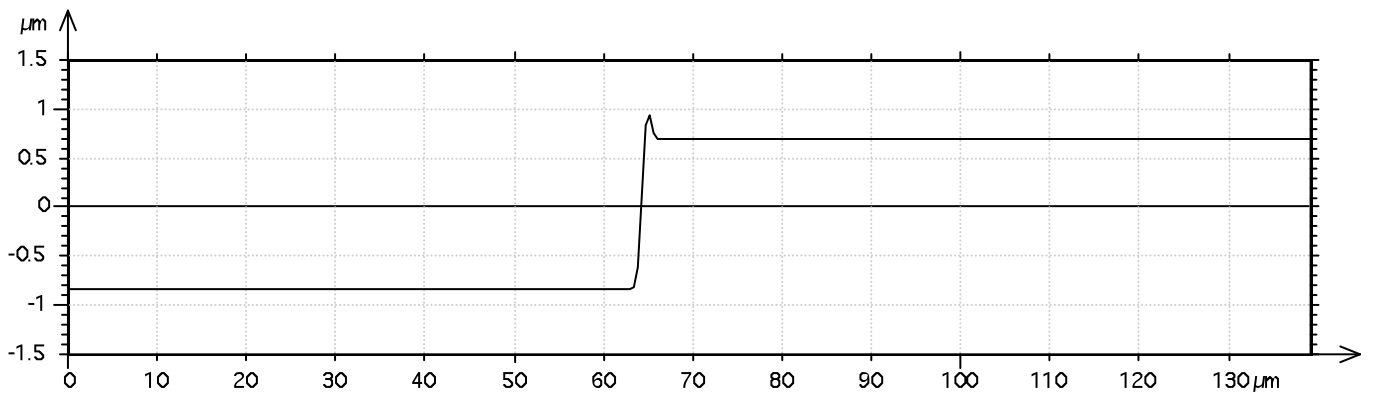

Figure 5. Anomalous result from Instrument B

It is interesting to note that this measurement appears to show a $2 \pi$ error at the edge discontinuity, however, in this case the edge is measured correctly but the upper surface is suppressed by $0.3 \mu \mathrm{m}$. The correct step height was reported when the step artefact was rotated 180 degrees and this suggests that the error is triggered by a mechanism similar to tilt 
dependent dispersion. Although the actual cause of the discrepancy is unknown, it suggests that Instrument B does not infer fringe order from the signal derived from each pixel but rather uses an algorithm that combines data from more that one pixel and in this case, has allowed the error to propagate.

\subsection{Measurement of sinusoidal profiles}

Two sets of sinusoidal artefact were measured by the SWLI instruments and the AFM. The objective of this work was to study the effects of surface gradient. For the most part, the instruments were tested using their high power, high numerical aperture objectives (50X NA $=0.55)$. The half-angle of the cone of light collected by these objectives is $\sin ^{-1}(0.55)=33.4$ degrees. As mentioned in Section 2 this provides an absolute limit to the surface gradient that can be measured.

Figure 6 shows the profile reported by Instrument D for the sinusoidal artefact with $25 \mu \mathrm{m}$ pitch and nominal peak-to-peak amplitude of $134 \mu \mathrm{m}$. It can be seen that the measurement is close to an ideal form and is typical of any of the instruments when measuring any of the low aspect ratio sinusoidal profiles (with a maximum gradient of 5 degrees or less).

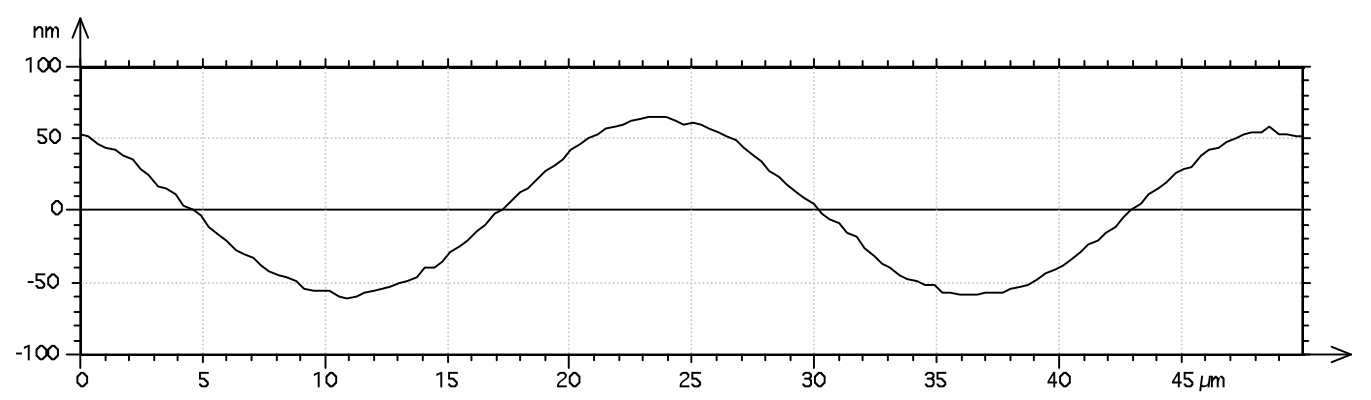

Figure 6. Profile obtained by Instrument D for the sinusoid of $25 \mu \mathrm{m}$ pitch, $134 \mu \mathrm{m}$ peak-topeak amplitude

In contrast, for the case of the high aspect ratio $25 \mu \mathrm{m}$ pitch sample with a nominal peakto-peak amplitude of $2.72 \mu \mathrm{m}$, all the SWLI instruments failed to achieve valid measurements around the area of maximum gradient. The profile of this sample measured with the AFM is shown in Figure 7. It is noted that this particular sample shows a significant departure from a ideal sinusoidal profile.

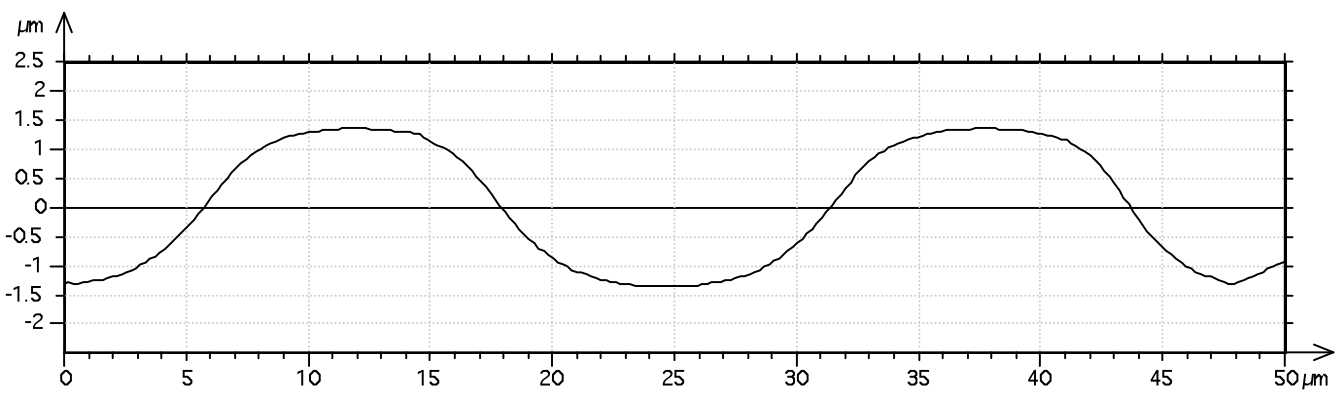

Figure 7. $25 \mu \mathrm{m}$ pitch, $2.72 \mu \mathrm{m}$ peak-to-peak amplitude sample measured with the AFM.

The results measured by the SWLI instruments are shown in Figure 8. Figure 8a shows the profile reported by Instrument A using the 50X objective (the Instrument B profile is similar). 


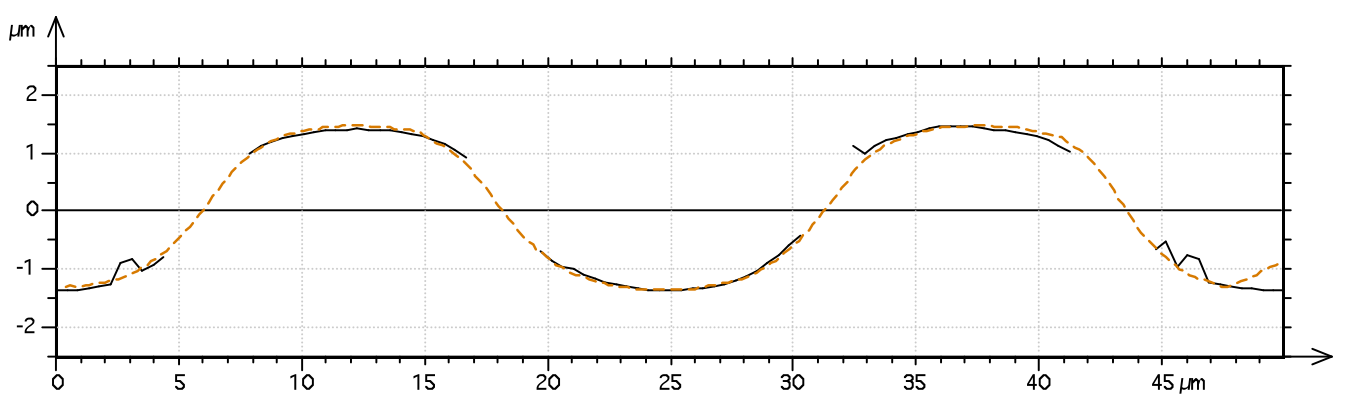

a) Instrument $A$

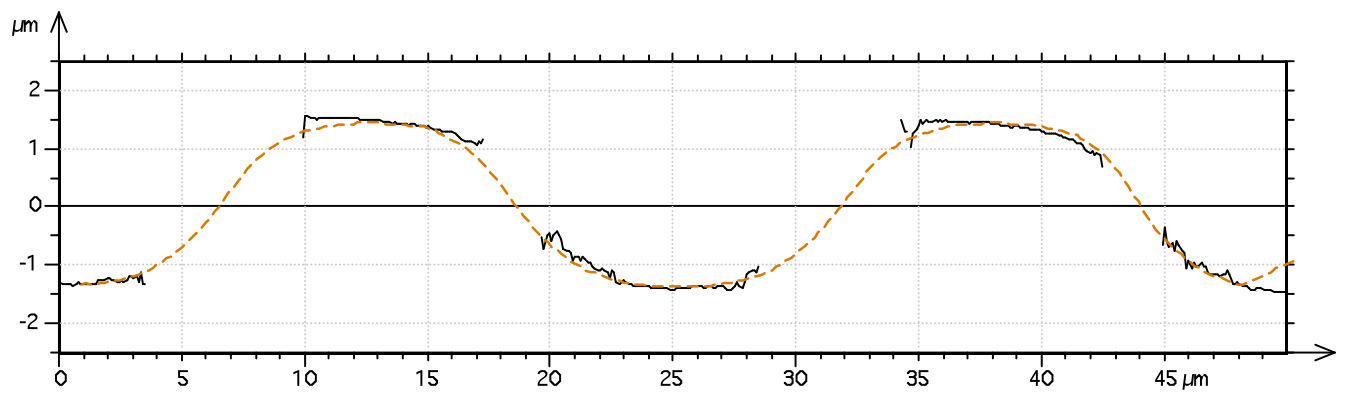

b) Instrument $C$

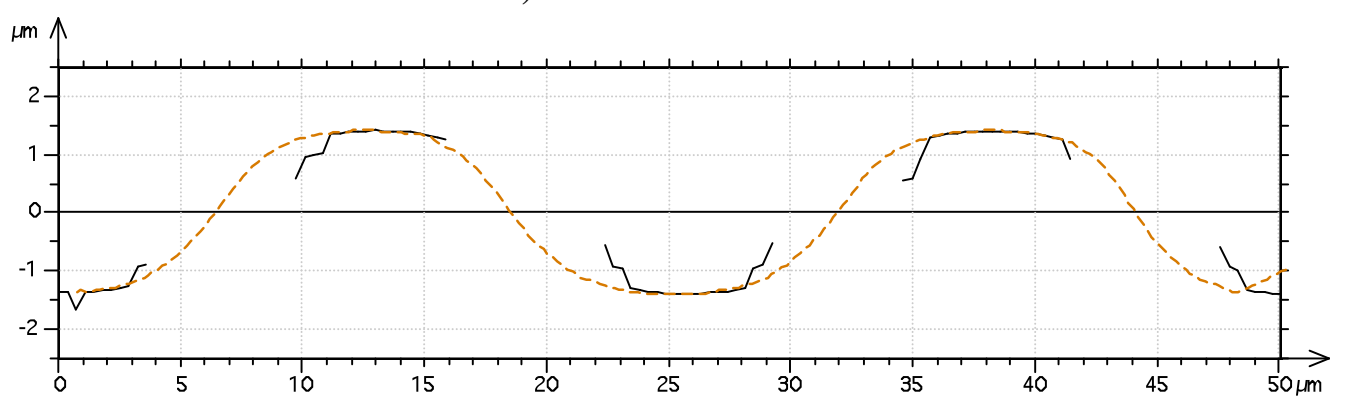

c) Instrument $D$

Figure 8. Measured profiles of a $25 \mu \mathrm{m}$ pitch, $2.72 \mu \mathrm{m}$ peak-to-peak amplitude sample

Clearly Instrument A is incapable of measuring the profile around the region of maximum gradient. For an ideal sinusoid with this amplitude the surface gradient is 18.8 degrees at this point and we would expect that the instruments would provide a valid measurement. From the AFM we note that this sample is not a good sinusoid and the surface gradient is significantly greater. We estimate that the maximum surface gradient is approximately 37 degrees. Of the SWLI instruments, Instrument A and Instrument B appear to be able to make measurements over the greatest range of surface gradients. We estimate that the maximum surface gradient that can be measured using these instruments is about \pm 22 degrees. The Instrument $C$ and Instrument $\mathrm{D}$ report data over a slightly smaller range that we estimate to be \pm 20 degrees. We note, however, that the response of Instrument $\mathrm{C}$ is asymmetric and provides better data over negative gradients (as presented in Figure 9c). This suggests that the instrument is not properly aligned.

It is also noted that there are small jumps in the reported profiles at well defined positions in each cycle. Individual jumps are most obvious in Instrument A and Instrument B measurements and correspond to half-wavelength, $2 \pi$ errors. The errors occur at positions where the surface gradient is approximately 17 degrees. At this angle approximately half of the illuminating light is collected by the objective and the difference in the ray paths between the returns from reference and object surface are most significant. These errors are likely to be caused by a tilt dependent dispersion as a result of chromatic aberration as discussed in Section 2. Finally, it is noted that Instrument D measures a more stepped profile than the 
other instruments. It appears that the steps are half-wavelength and can be considered as $2 \pi$ errors once again, however, these are steps rather than spikes and are most probably artefacts of the demodulation process. It appears that this instrument puts a greater emphasis on the fringe envelope than the phase of the fringes to make an estimate of surface height. It is likely that varying degrees of dispersion (as the surface gradient varies) would cause the instrument to partially "lock on" to the fringes as their position moves relative to the coherence envelope.

The profile obtained by the AFM for the sinusoidal artefact of $8 \mu \mathrm{m}$ pitch and $466 \mathrm{~nm}$ peak-to-peak amplitude is shown in Figure 9 and the corresponding results measured by the SWLI instruments are shown in Figure 10.

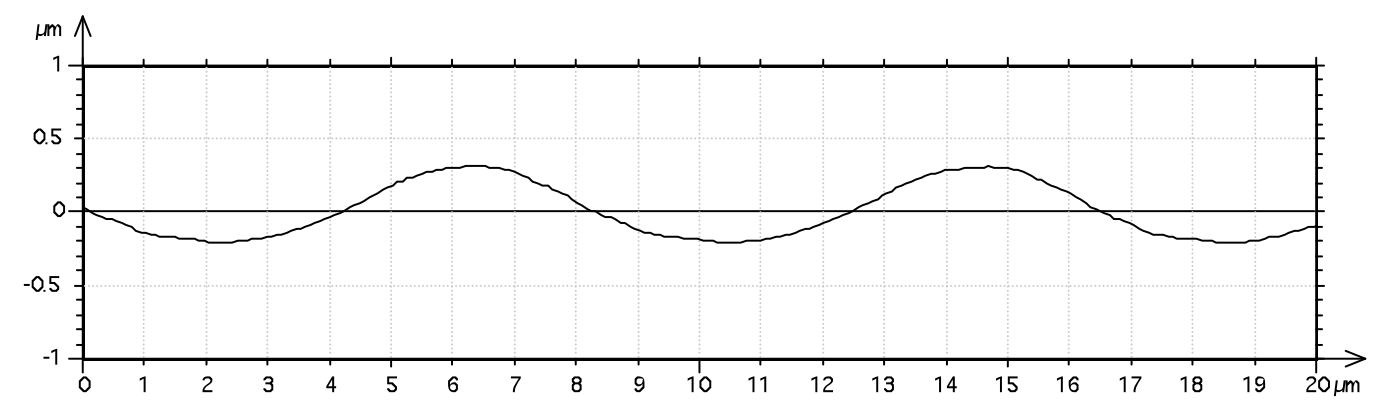

Figure $9.8 \mu \mathrm{m}$ pitch, $466 \mathrm{~nm}$ peak-to-peak amplitude sample measured with the AFM.

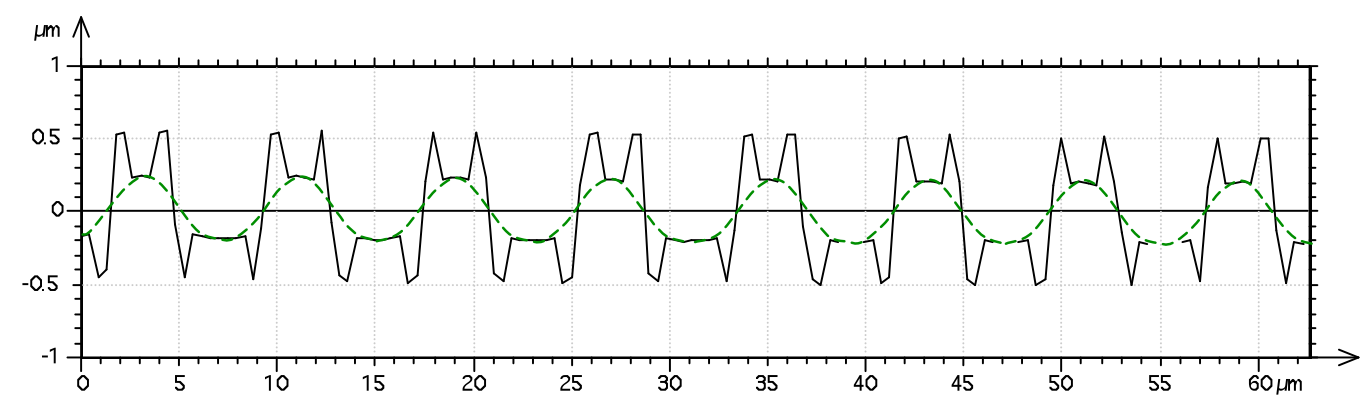

a) Instrument $\mathrm{A}$

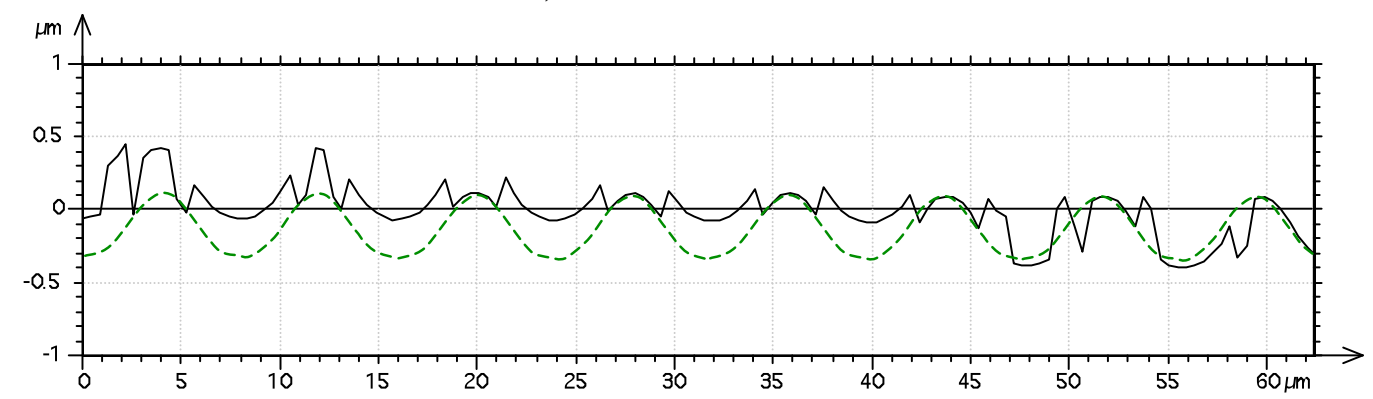

b) Instrument B

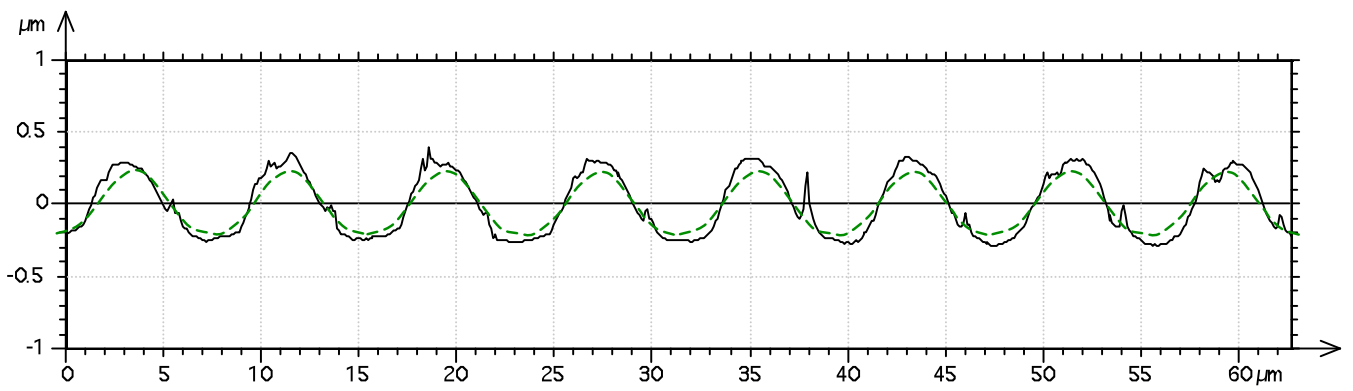

c) Instrument $C$ 


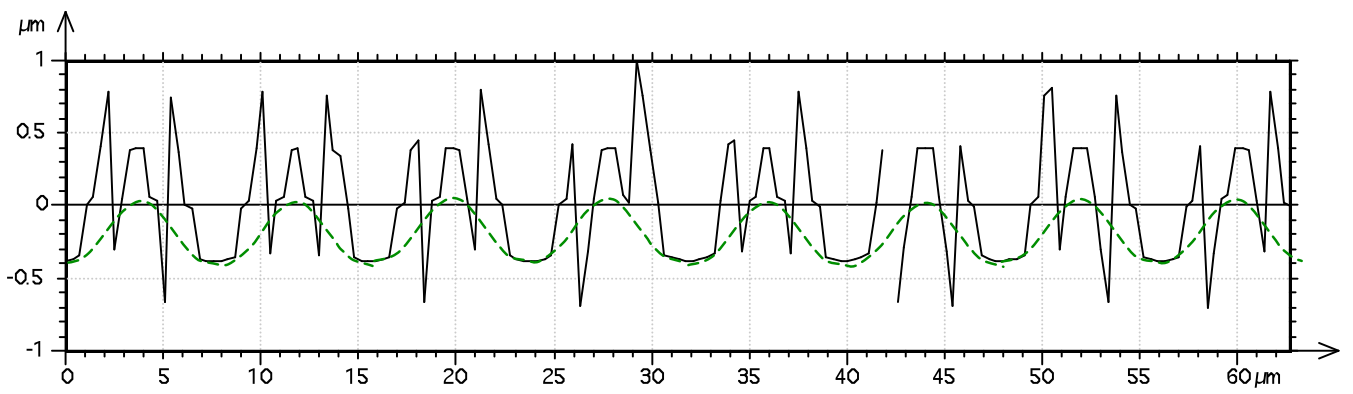

d) Instrument D

Figure $10.8 \mu \mathrm{m}$ pitch, $466 \mathrm{~nm}$ peak-to-peak amplitude sample measured with SWLI instrumentation

Figure 10a to 10d show the profiles reported by Instrument A, Instrument B, Instrument C and Instrument $\mathrm{D}$ respectively all using objectives of $\mathrm{NA}=0.55$. Here the maximum slope of the profile is approximately 17 degrees and this is substantially less than the acceptance angle of the objective. Of the SWLI instruments, Instrument $C$ appears to provide the best measurement. It is noted once again that this instrument is not working in VSI/PSI mode (see Section 2). Instrument A shows a fairly consistent result across the field although $2 \pi$ errors are clearly present. In this case the instrument reports the profile faithfully at the top and the bottom of the profiles where the surface gradient is within the range of \pm 11 degrees or so, but $2 \pi$ errors occur and a large step corresponding to a $6 \pi$ phase jump occurs. The Instrument $B$ shows a field dependent result that has frequent $2 \pi$ errors. The left hand side of this figure shows faithful reproduction of the peaks in the profile whereas the right hand side reports that of the troughs. The troughs on the left hand side are reproduced half a wavelength above their correct position and the peaks on the right hand side are a corresponding amount below their true position. Instrument D shows different characteristics. Here the instrument appears to report the troughs faithfully but the peaks are subject to $2 \pi$ errors. This error disappears as the magnitude of the surface gradient increases then reappears one more time before a full jump of $4 \pi$ radians restores a faithful measurement at the bottom of the groove.

From this data we conclude that $2 \pi$ errors are linked to surface gradient and are most probably caused by chromatic aberration leading to a tilt dependent dispersion. However, the points at which these errors occur also appear to depend on the grating pitch and errors are different in peaks and troughs. In other words errors depend on second order (and most probably higher order) statistics of the surface profile. Despite the frequency of $2 \pi$ errors the peak-to-peak amplitudes that are calculated from the Abbott curve (as described in Section 3) are for the most part consistent with that reported by the AFM. The results are presented in Table 4.

\begin{tabular}{|c|c|c|c|c|c|c|c|}
\hline \multirow[b]{2}{*}{$\begin{array}{l}\text { Sample } \\
\text { Pitch } \\
(\mu \mathrm{m})\end{array}$} & \multicolumn{7}{|c|}{ Peak-to-Peak Amplitude (nm) } \\
\hline & AFM & $\begin{array}{c}\text { Instrument } \\
\text { A } \\
(10 \mathrm{X})\end{array}$ & $\begin{array}{c}\text { Instrument } \\
\text { A } \\
(50 \mathrm{X})\end{array}$ & $\begin{array}{c}\text { Instrument } \\
\mathrm{B} \\
(50 \mathrm{X})\end{array}$ & $\begin{array}{c}\text { Instrument } \\
\mathrm{C} \\
(5 \mathrm{X})\end{array}$ & $\begin{array}{c}\text { Instrument } \\
\mathrm{C} \\
(50 \mathrm{X})\end{array}$ & $\begin{array}{c}\text { Instrument } \\
\text { D } \\
(50 \mathrm{X})\end{array}$ \\
\hline 25 & 2720 & 2830 & 2770 & 2780 & 2910 & 2790 & 2750 \\
\hline 25 & 654 & 916 & 772 & 717 & 818 & 786 & 706 \\
\hline 25 & 531 & 427 & 534 & 539 & 725 & 569 & 524 \\
\hline 25 & 134 & 56 & 126 & 129 & 302 & 154 & 127 \\
\hline 8 & 496 & 462 & 445 & 442 & 797 & 594 & 730 \\
\hline 8 & 124 & 43 & 91 & 104 & 333 & 178 & 107 \\
\hline 8 & 68 & 18 & 49 & 59 & 186 & 89 & 62 \\
\hline 8 & 20 & 6 & 20 & 20 & 40 & 20 & 21 \\
\hline
\end{tabular}


Table 4. Measurement results of sinusoidal gratings

A limited set of data was also obtained with lower power, smaller NA lenses on Instrument A (10X; NA = 0.30) and Instrument C $(5 \mathrm{X} ; \mathrm{NA}=0.12)$ and is shown for the case of the $25 \mu \mathrm{m}$ pitch, $2.72 \mu \mathrm{m}$ peak-to-peak amplitude sample in Figure 11a and $11 \mathrm{~b}$ respectively. For the case of Instrument A data is sparse as expected since the cone of maximum acceptance has a half-angle of approximately 17 degrees. However, Instrument $\mathrm{C}$ appears to measure the profile almost completely despite having an acceptance angle of only 6.9 degrees. It appears from this result that this instrument applies interpolation.

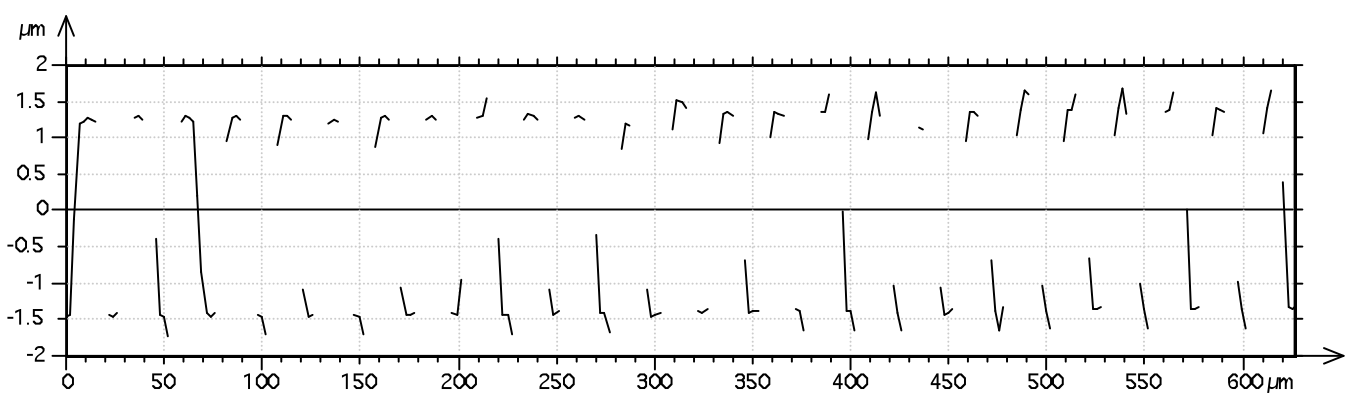

a) Instrument $A$

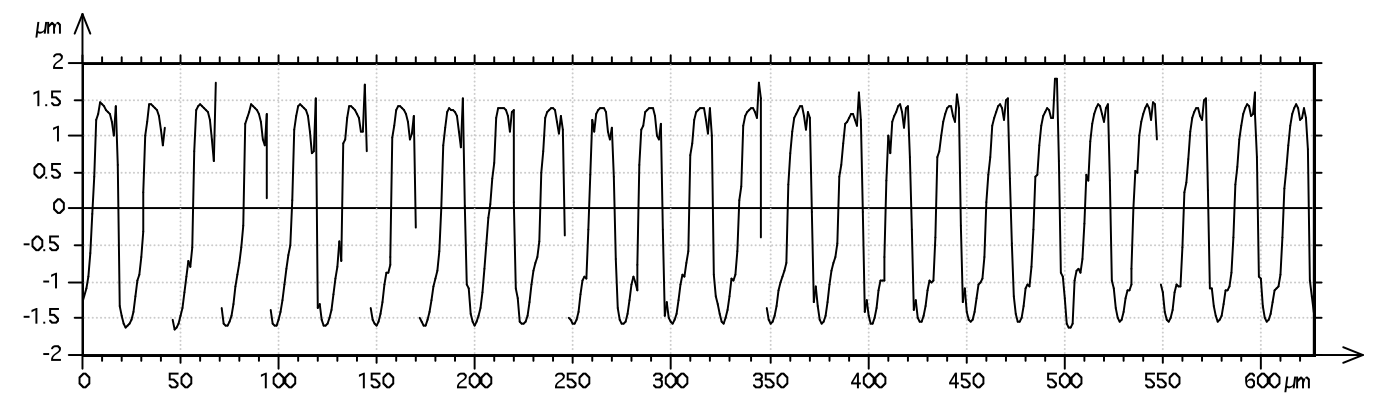

b) Instrument $C$

Figure 11. Measured profiles by low NA objective lenses

\subsection{Measurement on square grating samples}

To investigate the capability of the SWLI instruments in measuring the height of features approaching the lateral resolution of the objective, an etched silicon artefact consisting of a set of high spatial frequency rectangular profile gratings and a star pattern (as described in Section 3) was investigated. The etch depth was constant across the artefact with a nominal value of $184 \mathrm{~nm} \pm 1 \mathrm{~nm}$ as measured by the AFM. 


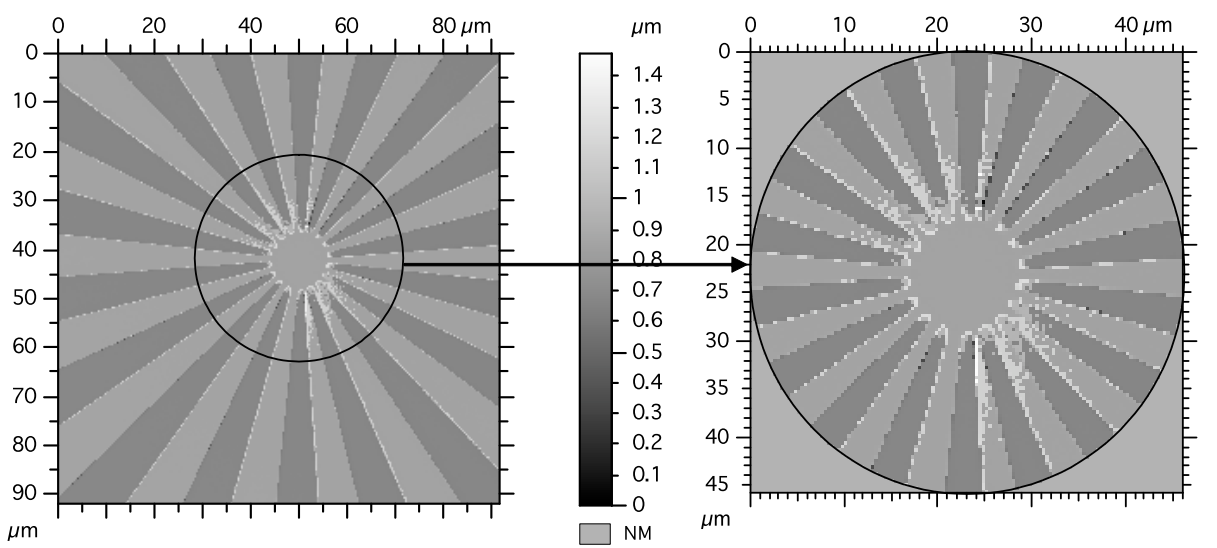

Figure 12. Measured image of a star pattern by Instrument D

The star pattern gives an idea of the SWLI capability over a range of spatial frequencies. A typical result in this case using Instrument D with the 50X objective is shown in Figure 12. For the most part it can be seen that the upper and lower surfaces are correctly located. However, $(2 \pi)$ errors can be seen at the step discontinuities and these increase toward the centre where the pitch is approximately $1.75 \mu \mathrm{m}$. It is also evident that the errors are not positioned symmetrically and there is greater probability of an error in the bottom right and top left quadrants. This is indicative of misalignment. It is interesting to note that measurements made with the $5 \mathrm{X}$ objective on Instrument $\mathrm{C}$ were surprisingly similar despite the instrument having a much smaller NA $=0.12$ and a lateral (Rayleigh) resolution of just $2.58 \mu \mathrm{m}$. Figure 13 shows this measurement.

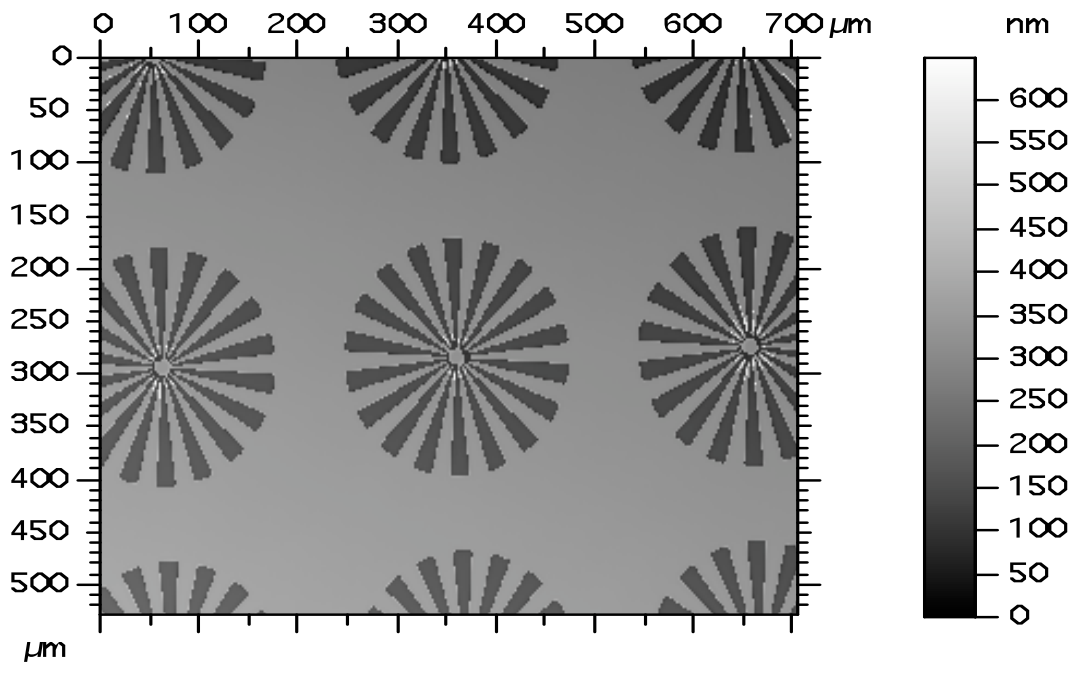

Figure 13. Measured image of star patterns by Instrument $C$

The set of rectangular profile gratings provides greater insight into the lateral resolution of the instruments. Figure 14a and 14b show typical profiles achieved by Instrument B with a 50X objective measuring gratings of $1 \mu \mathrm{m}$ and $2 \mu \mathrm{m}$ pitch respectively. For the $1 \mu \mathrm{m}$ pitch grating the measurement is swamped with $2 \pi$ errors. For the $2 \mu \mathrm{m}$ pitch the $2 \pi$ errors have largely disappeared and the rectangular profile is more apparent. Clearly the $2 \pi$ errors are not just edge artefacts but are affected to some extent by the local surface structure. It is interesting to note that Rayleigh resolution is approximately $0.67 \mu \mathrm{m}$ at a mean wavelength of $\lambda_{0}=600 \mathrm{~nm}$, and $\mathrm{NA}=0.55$ and consequently a microscope would be expected to easily resolve the $1 \mu \mathrm{m}$ grating. In this case the errors are most likely due to multiple scattering due to the sharp discontinuities and 90 degree internal angles that are within the coherence length of the illumination. 

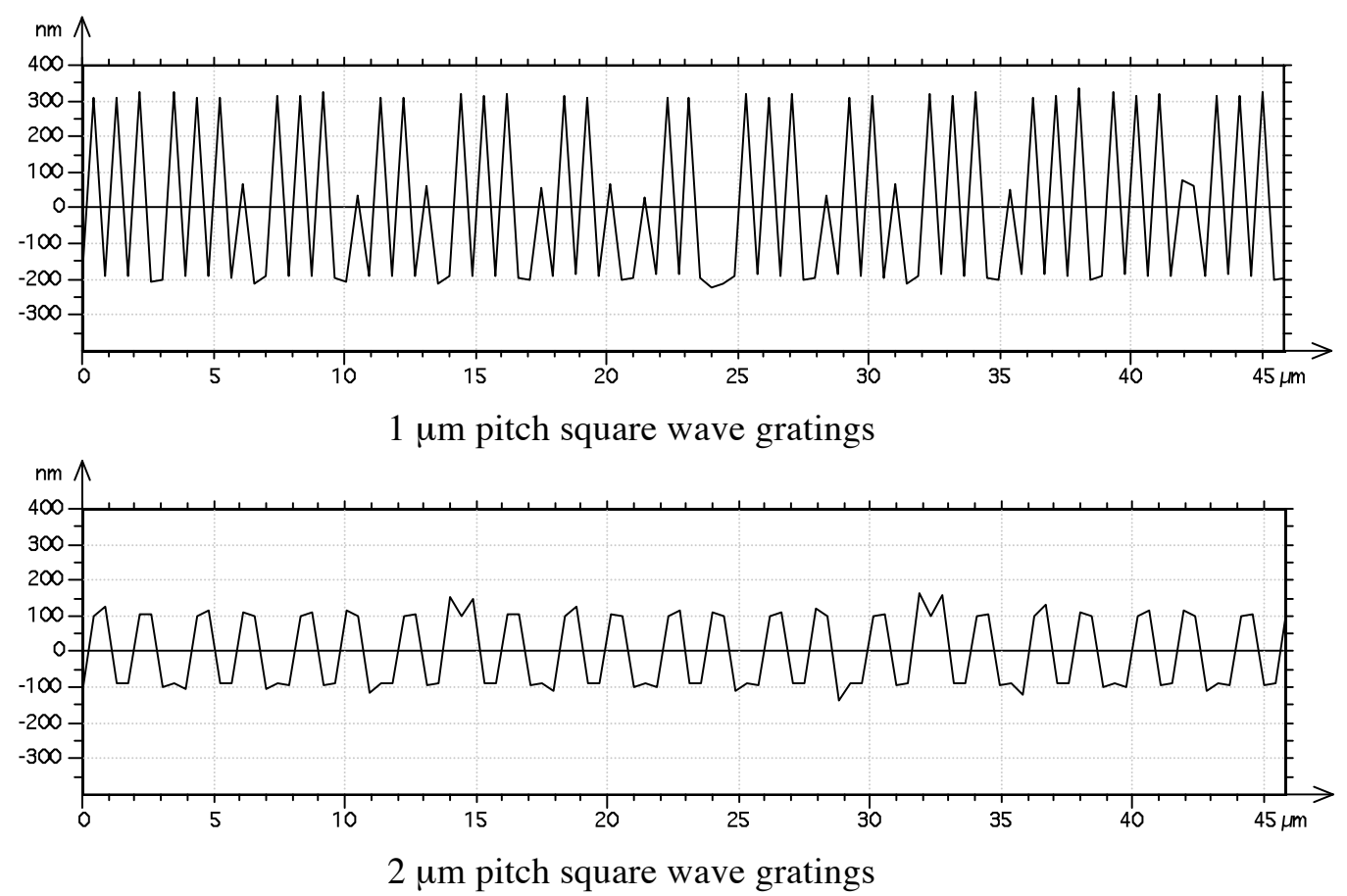

Figure 14. Measured profiles of $1 \mu \mathrm{m}$ and $2 \mu \mathrm{m}$ pitch square wave gratings by Instrument B

Similar results to Figure 14b were obtained with Instrument A, Instrument B and Instrument $\mathrm{D}$, however, Instrument $\mathrm{C}$ displayed batwing artefacts that confounded the measurements. Figure 15 shows a typical profile measured by this instrument.

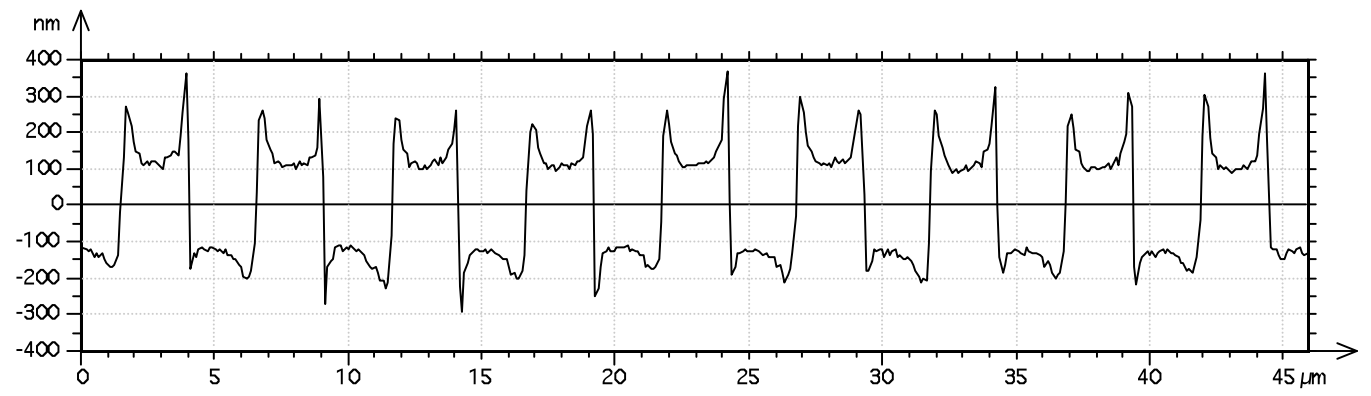

Figure 15. $5 \mu \mathrm{m}$ pitch Silicon artefact measured by Instrument $C$ with $50 \mathrm{X}$ objective lens

It can be seen that the batwing errors do not correspond to $2 \pi$ jumps and decay relatively slowly. In this case the peak-to-peak amplitude of the grating is overestimated. This characteristic, evaluated using the method described in Section 3, is summarised for the various instruments and artefacts in Table 5.

\begin{tabular}{cccccc}
\hline $\begin{array}{c}\text { Samples } \\
\text { Pitch }(\mu \mathrm{m})\end{array}$ & $\begin{array}{c}\text { AFM } \\
(\mathrm{nm})\end{array}$ & $\begin{array}{c}\text { Instrument A } \\
(\mathrm{nm})\end{array}$ & $\begin{array}{c}\text { Instrument B } \\
(\mathrm{nm})\end{array}$ & $\begin{array}{c}\text { Instrument C } \\
(\mathrm{nm})\end{array}$ & $\begin{array}{c}\text { Instrument } \mathrm{D} \\
(\mathrm{nm})\end{array}$ \\
\hline
\end{tabular}




\begin{tabular}{cccccc}
\hline 1 & 183 & 786 & 508 & 636 & 779 \\
2 & 185 & 194 & 194 & 475 & 194 \\
3 & 183 & 186 & 188 & 342 & 183 \\
4 & 185 & 185 & 185 & 263 & 184 \\
5 & 185 & 181 & 180 & 247 & 181 \\
6 & 185 & 180 & 181 & 243 & 181 \\
7 & 183 & 182 & 180 & 224 & 185 \\
8 & 186 & 184 & 180 & 217 & 183 \\
Star & 186 & 180 & 179 & 216 & 181 \\
\hline
\end{tabular}

Table 5. Measurement results of square wave and the star pattern samples by AFM, Instrument A, Instrument B, Instrument $\mathrm{C}$ and Instrument $\mathrm{D}$ with $50 \mathrm{X}$ objectives

It can be seen that the etch depth is faithfully measured by most of the instruments for the case of $2 \mu \mathrm{m}$ pitch gratings and above. However, Instrument $C$ consistently overestimates the etch depth for the reasons mentioned above. Once again we note that this instrument operates in VSI mode (see Section 2).

\section{Conclusions}

There is no doubt that SWLI provides fast areal surface measurement and can justifiably claim nanometre resolution in specific cases (such as step height measurement). The measurements presented in this paper show, however, that some care must be taken in the interpretation of measurements of more complex structures. It appears that the most frequently occurring errors are jumps or spikes of half the mean wavelength and these are reported more frequently as the surface gradient increases or when there is a step discontinuity. It is clear that these errors correspond to a mistake in the estimation of fringe order or equivalently a $2 \pi$ error in phase and are likely to be caused by dispersion (as a consequence of chromatic aberration). It was found that errors of this type were also prevalent at high spatial frequencies, where multiple scattering effects might be expected, and towards the edge of the field.

From the limited set of data presented here it appears that many of the errors are systematic and could potentially be reduced by applying some sort of compensation. If the dispersion characteristics of the objective could be mapped as a function of position and surface gradient, for example, then a model could be fitted to find the surface parameters that best explained the interference data. Further work is necessary, however, to ascertain what constraints such an approach would place on the surface measurement and what, if any, error this approach might introduce itself.

\section{Acknowledgements}

This paper was jointly funded by the EPSRC Grand Challenge Project "3D-Mintegration" (EP/E001904/1) and the National Measurement System Engineering Measurement Programme 2005 - 2008. Thanks are due to Mike Conroy (Taylor Hobson), Alex Winkel (Veeco) and Graeme Gibbons (Lambda Photometrics) for carrying out some of the measurements presented in this paper. We want to add acknowledgment of discussion with $\mathrm{P}$ de Groot and the others.

\section{References:}


[1] Flournoy P A, McClure R W, Wyntjes G 1972 White-light interferometric thickness gauge Appl. Opt. 11 1907-1915.

[2] Lee B S, Strand T C 1990 Profilometry with a coherence scanning microscope Appl. Opt. 29 3784-3788.

[3] Kino G S, Chim S S C 1990 Mirau correlation microscope Appl. Opt. 29 3775-3783.

[4] Dresel T, Haeusler G, Venzke H 1992 Three-dimensional sensing of rough surface by coherence radar Appl. Opt. 31 919-925.

[5] Deck L, de Groot P 1994 High-speed noncontact profiler based on scanning white light interferometry Appl. Opt. 33 7334-7338.

[6] de Groot P, Stoner R, de Lega X, 2006 Zygo Corp. US, Patent No. US7106454.

[7] Mansfield D 2006 The distorted helix: thin film extraction from scanning white light interferometry. Proc. SPIE, 6186618600

[8] Hillmann W 1990 Surface profiles obtained by means of optical methods-- are they true representations of the real surface Annals of the CIRP 39 581-583.

[9] Creath K 1989 Calibration of numerical aperture effects in interferometric microscope objectives Appl. Opt. 28 3333-3338.

[10] Harasaki A, Wyant J C 2000 Fringe modulation skewing effect in the white-light vertical scanning interferometry Appl. Opt. 39 2101-2106.

[11] Harasaki A, Schmit J, Wyant J C 2000 Improved vertical-scanning interferometry Appl. Opt. 39 2107-2115.

[12] Proertner A, Schwider J 2001 Dispersion error in white-light Linnik interferometers and its implications for evaluation procedures Appl. Opt. 40 6223-6228.

[13] Harasaki A, Schmit J, Wyant J C 2001 Offset of coherent envelope position due to phase change on reflection Appl. Opt. 40 2102-2106.

[14] de Groot P, de Lega X C, Kramer J, Turzhitsky M 2002 Determination of fringe order in white-light interference microscopy Appl. Opt. 41 4571-4578.

[15] Lehmann P 2003 Optical versus tactile geometry measurement - alternatives or counterparts. SPIE, 5144 183-196

[16] Lehmann P 2006 Systematic effects in coherence peak and phase evaluation of signals obtained with a vertical scanning white-light Mirau interferometer. Photonic Europe, Strasbourg, France, 3-7 April 6188

[17] Park M-C, Kim S-W 2001 Compensation of phase change on reflection in white-light interferometry for step height measurement Optics Letters 26 420-422.

[18] Rhee H, Vorburger T, Lee J, Fu J 2005 Discrepancies between roughness measurements obtained with phase-shifting and white-light interferometry Appl. Opt. 44 5919-5927.

[19] Brand U, Fluegge J 1998 Measurement capabilities of optical 3D-sensors for MST applications Microelectronic Engineering 41/42 623-626.

[20] Hillmann W, Brand U, Krystek M 1996 Capabilities and limitations of interference microscopy for two and three dimensional surface-measuring technology Measurement 19 95-102.

[21] Leach R K, Chetwynd D G, Blunt L, Haycocks J, Harris P, Jackson K, Oldfield S, Reilly S 2006 Recent advances in traceble nanoscale dimension and force metrology in the UK Meas. Sci. Technol. 17 467-476.

[22] Gao F, Coupland J, Petzing J 2006 V-groove measurements using white light interferometry. Photon06, Manchester, 4-7 September

[23] Cohen D K, Caber P J, Brophy C P, 1992 Wyko Corporation, US, Patent No. US5204734.

[24] de Groot P, 1995 Zygo Corp. US, Patent No. US5398113.

[25] Bankhead A D, 2003 Taylor Hobson Ltd. UK, Patent No. GB2385417. 